\title{
Analysis of diffraction efficiency of phase gratings in dependence of grooves number
}

\author{
A.Yu. Meshalkin ${ }^{1}$, C.S. Losmanschii ${ }^{1}$, V.O. Cazac $^{1}$, E.A. Achimova ${ }^{1}$, V.V. Podlipnov ${ }^{2,3}$ \\ ${ }^{1}$ Institute of Applied Physics, Academiei str. 5, MD-2028, Chisinau, Moldova \\ ${ }^{2}$ Samara National Research University, Moskovkoye Shosse 34, Samara, Russia, 443086 \\ ${ }^{3}$ Image Processing Systems Institute of RAS - Branch of the FSRC "Crystallography and \\ Photonics" RAS, Molodogvardejskaya street 151, Samara, Russia, 443001
}

\begin{abstract}
The analysis of dependence of diffraction efficiency on number of grooves of phase gratings with sinusoidal profile was performed by means of specially designed program. An Angular Spectrum method applied for monochromatic light propagation in far field through phase grating was used for calculation of diffraction efficiency of gratings. Diffraction efficiency maps of $0-5$ th diffraction orders were obtained for different number of grooves (from 0 to 1000). It is shown that changing the number of grooves makes it possible to tune smoothly the diffraction efficiency.
\end{abstract}

\section{Introduction}

Diffraction gratings are commonly used as dispersive elements in many optical systems. Applications include spectrometers, switching, tuning and trimming elements in dense wavelength-division multiplexing, visual display technology, external cavity lasers, etc. [1-3]. The diffraction efficiency of grating is an important parameter since it will strongly influence the final energy delivered by the optical diffraction system. High diffraction efficiency can be very challenging to achieve at the necessary spectral bandwidth in selected diffraction order [4]. To address these different challenges, several types of diffraction gratings can be used related either to the manufacturing process (ruled gratings, holographic gratings, master gratings, replica gratings), the nature of the modulation (surface relief gratings, volume gratings, amplitude gratings), or the optical configuration (transmission gratings, reflection gratings) [5].

The theoretical analysis of diffraction gratings is classically derived within the theory of scalar optics [6]. The diffraction grating is composed by a series of parallel and periodic ridges formed on the appropriate material [7]. The intensities of different diffraction orders can be analyzed by means of the Kirchhoff diffraction theory considering each ridge as a secondary source of radiation. By applying the Fraunhofer approximation, the discrete diffractive orders are given by the constructive interference position between the waves emitted by each source [8]. The diffracted intensity results from the product between the intensity function of a single ridge and the interference function. It classically features sharp peaks due to the interference function modulated by the intensity function. The former depends on the grating period while the latter depends on the geometry of the ridge $[9,10]$. However, as it is noted in [11], this simplified approach does not explain the relative intensities of the diffracted orders of a diffraction grating, because the diffraction envelope associated with the ridge shape and its width is ignored. Diffraction gratings with ridge width being half of the period are more common, but other relationships between period and ridge width are possible [12]. The effect of this 
envelope function is so important that some diffraction orders may disappear, although they fulfil the above-mentioned interference condition. For instance, the simplest binary diffraction grating, where the width of the ridge is equal to the half of the grating period, produces a diffraction pattern where all even diffraction orders are missing [13]. For optical processing and switching, the intensity ratio of the diffraction and main beams of the grating needs to be controlled to within a certain range. This ratio can be affected by the variation of duty cycle and phase depth.

In this work, we present an evaluation the diffracted order intensities of binary phase diffraction gratings with different duty cycles (defined as the ratio of the grating ridge width to the grating period) and ridge heights (depth of phase modulation). This method is based on Angular Spectrum method applied for monochromatic light propagation through phase grating. Both the duty cycle and ridge height value were taken in account since they are directly related to the diffraction order intensity. The proposed method allows the calculation of the intensity of the particular diffraction orders for both amplitude and phase gratings with arbitrary profile.

\section{Modelling of light diffraction by Angular Spectrum method}

Scalar approximations of phase or amplitude diffraction gratings can be derived on the basis of an electromagnetic theory starting with the Maxwell differential equations, while these can be developed into the methods of modern Fourier optics. In the object and image plane, small patches surrounding the analyzed point were considered and associate them with the Fourier expansion, a superposition of plane waves [14]. The concept of intensity is tightly related to space-frequency distribution in the prediction of the phase morphology of microstructures. It was shown in [15] that free propagation of light with imaging of diffraction pattern can be modeled with good accuracy by computing the propagation of a complex wave, which can be done using several approximations [16]. For simulating of the diffraction process we applied Angular Spectrum method (AS) [17] that involves expanding a complex wave into a summation of infinite number of plane waves, and is suitable for both near and far field of diffraction [18]. In far field the AS method recovers Fraunhofer diffraction that is used for diffraction efficiency analyses.

Let us suppose the coherent light is being transmitted through the phase diffraction grating. Monochromatic beam of the plane waves falls normally to the grating.

The angular spectrum of the diffracted light $F_{0}\left(k_{x}, k_{y}\right)$ is described by the expression:

$$
F_{0}\left(k_{x}, k_{y}\right)=\frac{1}{(2 \pi)^{2}} \iint_{-\infty}^{\infty} u_{0}(x, y, 0) \exp \left[-\mathrm{i}\left(k_{x} x+k_{y} y\right)\right] \mathrm{d} x \mathrm{~d} y .
$$

The complex amplitude of the wave in the plane $z=0$ is described by the function $\mathrm{u}_{0}(\mathrm{x}, \mathrm{y}, 0)$.

In fact, in the plane $z=0$ the integrand of (1) expresses the complex amplitude of the plane harmonious wave with the components of the wave vector $\mathrm{k}_{\mathrm{x}}, \mathrm{k}_{\mathrm{y}}$ and $\mathrm{k}_{\mathrm{z}}$

$$
k_{z}=\sqrt{k^{2}-k_{x}^{2}-k_{y}^{2}}
$$

The complex spectrum $\mathrm{F}_{0}\left(\mathrm{k}_{\mathrm{x}}, \mathrm{k}_{\mathrm{y}}\right)$ depends upon the distribution of direction (time-term multiplier $\exp (-\mathrm{i} \omega \mathrm{t})$ is omitted). Variables $\mathrm{k}_{\mathrm{x}}=\mathrm{k} \cos \alpha, \mathrm{k}_{\mathrm{y}}=\mathrm{k} \cos \beta, \mathrm{kz} \beta=\mathrm{k} \cos \varphi$, where $\cos \alpha, \cos \beta, \cos \varphi$ are the direction cosines of the wavefront normal and $k=2 \pi / \lambda$ is the wave number. The initial distortion of the angular spectrum depends upon the range of $\mathrm{k}_{\mathrm{x}}$ and $\mathrm{k}_{\mathrm{y}}$, the width of the angular spectrum $\mathrm{F}_{0}\left(\mathrm{k}_{\mathrm{x}}, \mathrm{k}_{\mathrm{y}}\right)$ in the plane $z=0$. The transmission characteristic of the grating $T(x, y)$ was introduced. Every particular grating is characterized by its own $\mathrm{T}(\mathrm{x}, \mathrm{y})$.

If we define the field of the falling wave in the plane $z=0$ as $u_{f}(x, y)$, then the field behind the grating is defined by the formula $\mathrm{u}_{0}(\mathrm{x} ; \mathrm{y} ; 0)=\mathrm{u}_{\mathrm{f}}(\mathrm{x} ; \mathrm{y}) * \mathrm{~T}(\mathrm{x}, \mathrm{y})$ and the angular spectrum behind the grating is equal to the convolution of the angular spectrum of the falling field and the spectrum of the transmission grating.

In the case of phase grating diffraction, shown in Fig. 1., the grating characteristic function $T(x, y)$ is equal to $\mathrm{T}(\mathrm{x}, \mathrm{y})=\mathrm{A}_{0} \exp (\mathrm{i} \Delta \varphi(\mathrm{x}, \mathrm{y}))$, where $\mathrm{A}_{0}$ - is constant amplitude, and $\Delta \varphi(\mathrm{x}, \mathrm{y})$ - is light phase modulation induced by grating. Diffraction pattern from phase grating, that produces specified light phase modulation was obtained by AS in the case of unit amplitude $(\mathrm{A} 0=1)$ and distance $\mathrm{z}=10 \mathrm{~mm}>>\lambda$ 
(far field diffraction). The intensity of each diffraction order $I_{m}$ was collected and the diffraction efficiency was calculated by $\eta_{m}=I_{m} / I_{0} * 100 \%$, where $I_{0}=A_{0}{ }^{2}$ - is the intensity of incident light, $m$ diffraction order. In our simulation we used $\lambda=650 \mathrm{~nm}$.

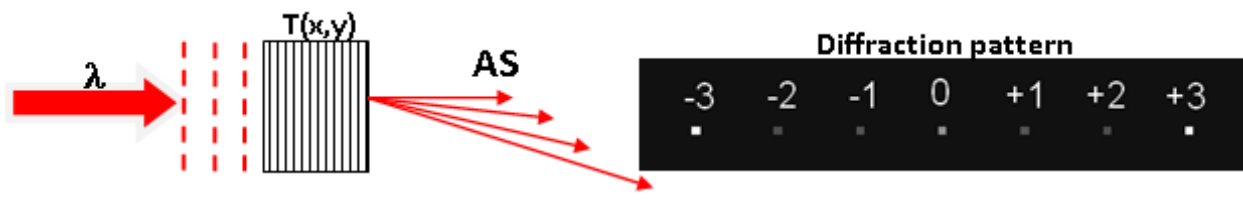

Figure 1. Diffraction on phase grating described by function $T(x, y)$ and imaging of diffraction pattern using AS.

\section{Diffraction from phase grating with different number of grooves}

The schematic of a diffraction from phase grating with rectangular profile is shown in Fig. 2, where $a$ is ridge width, $b$ is grating period, and $h$ is relief depth. The grating duty cycle parameter (D) is defined as the ratio of the grating ridge width to the period $\mathrm{D}=\mathrm{a} / \mathrm{b} * 100 \%$. The grating period was considered of being constant with value of $5 \mu \mathrm{m}$, while the ridge with was varied from $0 \mu \mathrm{m}$ to $5 \mu \mathrm{m}$ resulting in $\mathrm{D}$ variation from $0 \%$ up to $100 \%$.

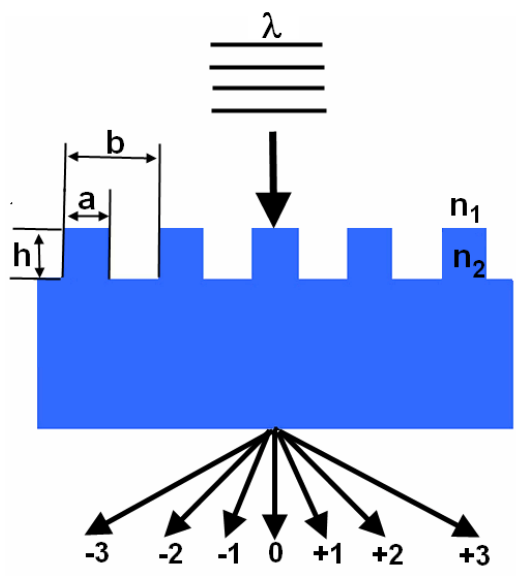

Figure 2. Schematic representation of light diffraction from phase grating with rectangular profile $(\lambda$ wavelength of incident plane wave, $\mathrm{n} 1$ and $\mathrm{n}_{2}$ - refractive indexes of surrounding media and grating correspondingly, $\mathrm{a}$ - bridge width; $\mathrm{b}$ - grating period, $\mathrm{h}$ - grating depth, $-3,-2, \ldots,+3$ - corresponding diffraction orders).

A plane wave illuminates the grating under normal incidence. The phase of light that is passed through such grating is modulated according to the grating profiley $\Delta \varphi(\mathrm{x}, \mathrm{y}) \sim \mathrm{h}(\mathrm{x}, \mathrm{y})$. The amplitude of phase modulation $\Delta \varphi$ induced by surface relief grating can be expressed as $\Delta \varphi=2 \pi / \lambda\left(\mathrm{h}^{*}\left(\mathrm{n}_{2}-1\right)\right)$ (3), and by refractive index grating as $\Delta \varphi=2 \pi / \lambda\left(\mathrm{d}^{*} \Delta \mathrm{n}\right)$ (4), where $\lambda$ is the light wavelength, $\mathrm{h}$ - surface modulation depth, $\mathrm{n}_{2}$ - refractive index of grating material, $\mathrm{d}$ - film thickness, $\Delta \mathrm{n}$ - amplitude of refractive index modulation [7]. A duty cycle parameter was varied from $0 \%$ to $100 \%$ by step $1 \%$.

\section{Conclusions}

The analysis of diffraction efficiency on duty cycle and light modulation induced by phase gratings with rectangular and Gaussian profile is described. Diffraction efficiency maps of $0-5^{\text {th }}$ diffraction orders were obtained for each type of grating profile. It was established missing of some diffraction orders for certain duty cycles of gratings with rectangular profile. The dependence of the diffraction efficiency on duty cycle is symmetrical relative to varying of duty cycle value for the grating with rectangular profile, while the same dependence of diffraction efficiency for grating with Gaussian profile is asymmetrical: maximum diffraction efficiency value shifts to higher values of duty cycle and reaches peak value at $\mathrm{D}=62.5 \%$. At this value the profile of grating is close to sinusoidal. Changing the duty cycle of grating makes it possible to tune smoothly the diffraction efficiency and to redirect the light intensity in required diffracted orders. 
Секция: Компьютерная оптика и нанофотоника

Analysis of diffraction efficiency of phase gratings in dependence of grooves number

\section{Acknowledgments}

This research was partially supported by the H2020-TWINN-2015 HOLO project (nr. 687328), Russian Foundation for the Basic Research (Project No 18-29-20045-мк, Project No 16-29-09528 ofim, Project No. 18-07-01470).

\section{References}

[1] Bonod, N. Diffraction gratings: from principles to applications in high-intensity lasers / N. Bonod, J. // Neauport, Adv. Opt. Photon. - 2016. - Vol. 8. - P. 156-199.

[2] Alonso-Ramos, C. Polarization-independent grating coupler for micrometric silicon rib waveguides / C. Alonso-Ramos, L. Zavargo-Peche, A. Ortega-Moñux, R. Halir, I. MolinaFernández, P. Cheben // Optics Letters. - 2012. - Vol. 37(17). - P. 3663-3665.

[3] Feng, J. Three-port beam splitter of a binary fused-silica grating / J. Feng, C. Zhou, B. Wang, J. Zheng, W. Jia, H. Cao, P. Lv // Appl. Opt. - 2008. - Vol. 47. - P. 6638-6643.

[4] Rathgen, H. Large bandwidth, highly efficient optical gratings through high index materials / H. Rathgen, H.L. Offerhaus // Opt. Express. - 2009. - Vol. 17. - P. 4268-4283.

[5] Palmer, C. Diffraction Grating Handbook - Richardson Gratings, Newport Corporation, 2014.

[6] Andries, I. Approximate analysis of the diffraction efficiency of transmission phase holographic gratings with smooth non-sinusoidal relief / I. Andries, T. Galstian, A. Chirita // J. Optoel. Adv. Mater. - 2016. - Vol. 18. - P. 56-64.

[7] Cazac, V. Surface relief and refractive index gratings patterned in chalcogenide glasses and studied by off-axis digital holography / V. Cazac, A. Meshalkin, E. Achimova, V. Abashkin, V. Katkovnik, I. Shevkunov, D. Claus, G. Pedrini // Appl. Opt. - 2018. - Vol. 57. - P. 507-513.

[8] Duffieux, P.M. The Fourier Transform and its Applications to Optics - Wiley, New York, 1983.

[9] Ustinov, A.V. Effect of the fill factor of an annular diffraction grating on the energy distribution in the focal plane / A.V. Ustinov, A.P. Porfir'ev, S.N. Khonina // J. Opt. Technol. - 2017. - Vol. 84. - P. 580-587.

[10] Khonina, S.N. Diffractive axicon with tunable fill factor for focal ring splitting / S.N. Khonina, A.P. Porfirev, A.V. Ustinov // Proc. SPIE. - 2017. - Vol. 10233. - P. 102331P.

[11] Mezouari, S. Validity of Fresnel and Fraunhofer approximations in scalar diffraction / S. Mezouari, A.R. Harvey // Journal of Optics A: Pure and Applied Optics. - 2003. - Vol. 5(4). P. S86-S91.

[12] Torcal-Milla, F.J. Diffraction by gratings with random fill factor / F.J. Torcal-Milla, L.M. Sanchez-Brea // Appl. Opt. - 2017. - Vol. 56. - P. 5253-5257.

[13] Shih, W.-C. High-resolution electrostatic analog tunable grating with a single-mask fabrication process / W.-C. Shih, S.-G. Kim, G. Barbastathis // Journal of Microelectromechanical Systems. - 2006. - Vol. 15(4). - P. 763-769.

[14] Comastri, S.A. Generalized sine condition for image-forming systems with centering errors / S.A. Comastri, J.M. Simon, R. Blendowske // J. Opt. Soc. Am. A. - 1999. - Vol. 16(3). - P. 602-612.

[15] Matsushima, K. Band-Limited Angular Spectrum Method for Numerical Simulation of FreeSpace Propagation in Far and Near Fields / K. Matsushima, T. Shimobaba // Opt. Express. 2009. - Vol. 17. - P. 19662-19673.

[16] Kreis, T.M. Methods of digital holography: A comparison, in Optical Inspection and Micromeasurements II / T.M. Kreis, M. Adams, W.P.O. Juptner // Proc. SPIE . - 1997. - Vol. 3098. - P. 224-233.

[17] Shimobaba, T. Scaled angular spectrum method / T. Shimobaba, K. Matsushima, T. Kakue, N. Masuda, T. Ito // Opt. Lett. - 2012. - Vol. 37. - P. 4128-4130.

[18] Kozacki, T. Angular spectrum-based wave-propagation method with compact space bandwidth for large propagation distances / T. Kozacki, K. Falaggis // Opt. Lett. - 2015. - Vol. 40. - P. $3420-3423$.

[19] Goodman, J.E. Introduction to Fourier Optics - New York: McGraw-Hill, 1996. 